\title{
A Statistical Sampling Strategy for Iris Recognition
}

\author{
Luis E. Garza Castañon ${ }^{1}$, Saul Montes de Oca², and \\ Rubén Morales-Menéndez ${ }^{1}$ \\ 1 Department of Mechatronics and Automation, ITESM Monterrey Campus, \\ $\{$ legarza,rmm\}@itesm.mx \\ 2 Automation Graduate Program Student, ITESM Monterrey Campus, \\ saul_montesdeoca@yahoo.com.mx. \\ Av. Eugenio Garza Sada Sur No. 2501 \\ Monterrey, N.L. 64849 México
}

\begin{abstract}
We present a new approach for iris recognition based on a random sampling strategy. Iris recognition is a method to identify individuals, based on the analysis of the eye iris. This technique has received a great deal of attention lately, mainly due to iris unique characterics: highly randomized appearance and impossibility to alter its features. A typical iris recognition system is composed of four phases: image acquisition and preprocessing, iris localization and extraction, iris features characterization, and comparison and matching. Our work uses standard integrodifferential operators to locate the iris. Then, we process iris image with histogram equalization to compensate for illumination variations. The characterization of iris features is performed by using accumulated histograms. These histograms are built from randomly selected subimages of iris. After that, a comparison is made between accumulated histograms of couples of iris samples, and a decision is taken based on their differences and on a threshold calculated experimentally. We ran experiments with a database of 210 iris, extracted from 70 individuals, and found a rate of succesful identifications in the order of $97 \%$.
\end{abstract}

\section{INTRODUCTION}

Iris recognition is an specific area of biometrics. The main intention of biometrics is to provide reliable automatic recognition of individuals based on the measuring of a physical characteristic or personal trait. Biometrics can be used for access control to restricted areas, such as airports or military installations, access to personal equipments such as laptops and cellular phones, and public applications, such as banking operations [11]. A wide variety of biometrics systems have been explored and implemented with different degrees of success. Resulting systems include different physiological and/or behavioral human features such as: gait, DNA, ear, face, facial thermogram, hand thermogram, hand vein, fingerprint, hand shape, palmprint, signature, voice and iris $[7,8]$. The last one may provide the best solution by offering a much more discriminating power than others 
biometrics. Specific characteristics of iris such as a data-rich structure, genetic independence, stability over time and physical protection, makes the use of iris as biometric well recognized.

In last years, there have been very successful implementations of iris recognition systems. Differences between them are mainly in the features characterization step. The golden standard is set by Daugman's system, with a performance of $99.9 \%$ of matching [3]. Daugman used multiscale quadrature wavelets (Gabor filters) to extract texture phase structure information of the iris to generate a 2,048-bit iriscode and compared the difference between a pair of iris representations by their Hamming distance. In [10] iris features are extracted by applying a dyadic wavelet transform with null intersections. To characterize the texture of the iris, Boles and Boashash [1] calculated a one dimension wavelet transform at various resolution levels of a concentric circle on an iris image. In this case the iris matching step was based on two dissimilarity functions. Wildes [13] represented the iris texture with a Laplacian pyramid constructed with four different resolution levels and used the normalized correlation to determine whether the input image and the model image are from the same class. A Similar method to Daugman's is reported in [9], but using edge detection approach to localize the iris, and techniques to deal with illumination variations, such as histogram equalization and feature characterization by average absolute deviation. In [5] iris features are extracted by using independent component analysis. Zhu et al [14] uses statistical features, mean and standard deviations, from 2D wavelets transforms and Gabor filters, to make the system more robust to rotation, translation and illumination variations of images. In [6] a new method is presented to remove noise in iris images, such as eyelashes, pupil, eyelids and reflections. The approach is based on the fusion of edge and region information. In [4] an iris recognition approach based on mutual information is developed. In this work couples of iris samples are geometrically aligned by maximizing their mutual information and subsequently recognized. The mutual information was calculated with the algorithm proposed by Darbellay and Vajda. The decision whether two compared images belong to the same eye depends on a chosen threshold of mutual information.

In our approach we work directly with the iris information, instead of using a bank of filters or making a mathematical transformation. These approaches can be very computationally demanding. We claim than our approach will conduct to a fast approach for iris recognition, where just a few samples will be needed to discard many samples in database and allow a more focused sampling in a reduced set of candidate samples.

In our work, a database with colored high resolution eye images, is processed to lower the size and transform to a grey levels image. After this, the iris is located by using integrodifferential operators and extracted by using a transformation from cartesian to polar coordinates. The result of this operation is a rectangular strip containing just the iris area features. To compensate for illumination variations, iris strip is processed by histogram equalization. The feature extraction step is done by randomly sampling square subimages, and building 
an acummulated histogram for each subimage. Every iris is represented by a set of accumulated histograms. The optimal size of square areas and the number of features were calculated experimentally. The comparison between iris sample and database is done by computing the Euclidean distance between histograms, and according to a threshold calculated also experimentally, we take the decision to accept o reject the iris sample.

We ran experiments in a database containing 210 samples coming from 70 individuals and found a rate of succesful matching in the order of $97 \%$.

\section{THE PROPOSED APPROACH}

The implementation of our approach relies on the use of eyes images from a database. These images use a format with color and high resolution, which can give us some problems with the management of memory. In our database, images can contain more than 6 Mbytes of information. Then, the first step consists in down sizing the image (we use $1024 \times 758$ bytes), and transform from color representation to just grey level pixels. This process is sufficient to reveal the relevant features of iris. Eyes images include samples where iris is free from any occlusion, as is shown in figure 1, and others with moderate obstruction from eyelids and eyelashes (Fig. 2).

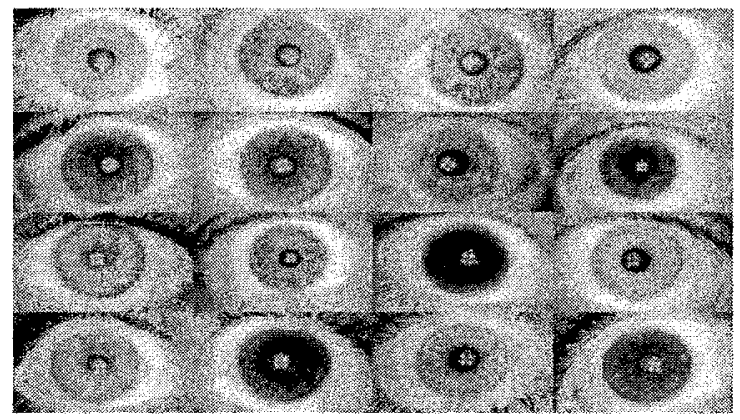

Fig. 1. Eyes samples without noise

\subsection{Iris Localization}

The finding of limbic and pupilar limits is achieved with the use of the standard integrodifferential operator shown in eqn 1.

$$
\left(r, x_{0}, y_{0}\right)=\left|\frac{\partial}{\partial r} G(r) * \oint_{r, x_{c}, y_{c}} \frac{I(x, y)}{2 \pi r} d s\right|
$$




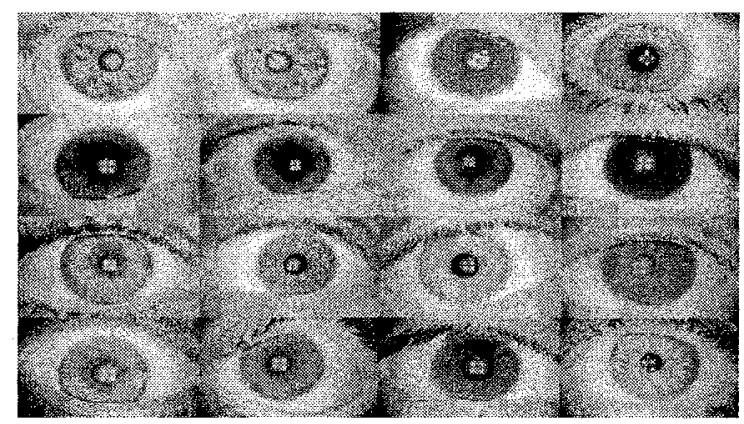

Fig. 2. Eyes samples with noise (moderate obstruction)

where $I(x, y)$ is an image containing an eye.

The operator behaves as an iterative circular edge detector, and searches over the image domain $(x, y)$ for the maximum in the partial derivative with respect to an increasing radius $r$, of the normalized contour integral of $I(x, y)$ along a circular are $d s$ of radius $r$ and center coordinates $\left(x_{0}, y_{0}\right)$. The symbol $*$ denotes convolution and $G_{\sigma}(r)$ is a smoothimg function, tipically a Gaussian of scale $\sigma$.

This operator behaves well in most cases with moderate noise conditions, but requires some fine tuning of parameters, in order to deal with pupil reflections, obscure eyes and excess of illumination. Heavy occlusion (iris area covered more than $40 \%$ ) of iris by eyelashes or eyelids needs to be handled by other methods. The extracted iris image has to be normalized to compensate for pupil dilation and contraction under illumination variations. This process is achieved by a transformation from cartesian to polar coordinates, using equations 2 and 3 . The output of this transformation is a rectangular image strip, shown in Fig. $5(\mathrm{a})$.

$$
\begin{aligned}
& x(r, \theta)=(1-r) x_{p}(\theta)+r x_{s}(\theta) \\
& y(r, \theta)=(1-r) y_{p}(\theta)+r y_{s}(\theta)
\end{aligned}
$$

where $x(r, \theta)$ and $y(r, \theta)$ are defined as a linear combination of pupil limits $\left(x_{p}(\theta), y_{p}(\theta)\right)$ and limbic limits $\left(x_{s}(\theta), y_{s}(\theta)\right), r$ is defined in the interval $[0,1]$, and $\theta$ in the interval $[0,2 \pi]$.

\subsection{Features Extraction}

The iris image strip obtained in previous step, is processed by using an histogram equalization method, to compensate for differences in illumination conditions.

The main objective in this method is that all grey levels (ranging from 0 to 255) have the same number of pixels. Histogram equalization is obtained by working with the accumulated histogram, shown in eqn 4 . 


$$
H(i)=\sum_{k=0}^{i} h(k)
$$

where $h(k)$ is the histogram of the $k t h$ grey level.

A flat histogram, where every grey level has the same number of pixels, can be obtained by eqn 5 .

$$
G\left(i^{\prime}\right)=\left(i^{\prime}+1\right) \frac{N * M}{256}
$$

Where $N$ and $M$ are the image dimensions and 256 is the number of grey levels. An example of application of histogram equalization method over the iris strip in figure $5(\mathrm{a})$, is shown in figure $5(\mathrm{~b})$.
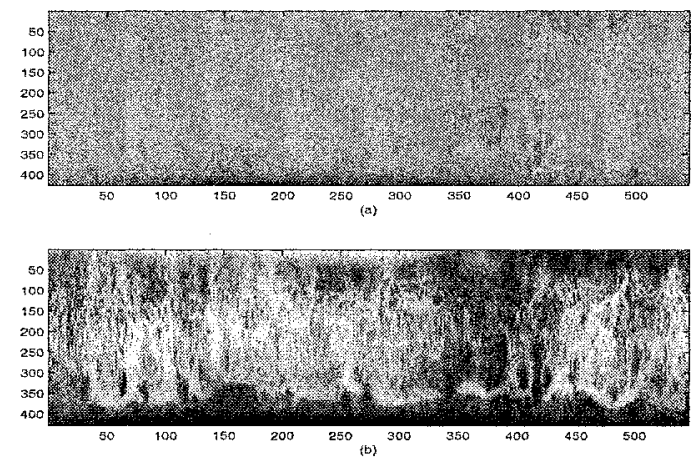

Fig. 3. (a) Extracted iris strip image.(b) Iris strip processed by the histogram equalization method

\subsection{Comparison and Matching}

In our method, the iris features are represented by a set of accumulated histograms computed from randomly selected square subimages of iris strip (see Fig. 6). An accumulated histogram represents a feature and is built by using equation 4 . The complete iris is represented by a set of accumulated histograms, one of them for every subimage. The optimal size of the number of features and subimage size, were determined empirically by experiments. A decision to accept or reject the iris sample is done according to the minimum Euclidean distance calculated from the comparison of iris sample and irises database, and also according to a threshold. Figure 8 shows the structure of this phase. 


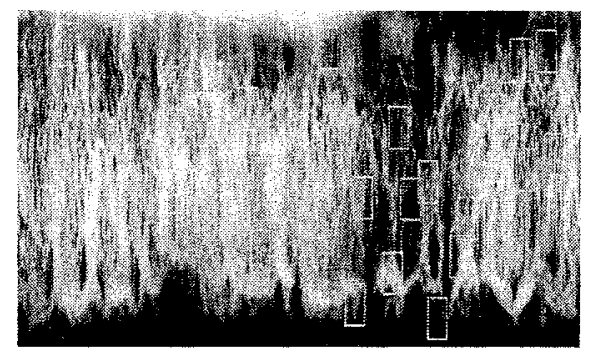

Fig. 4. iris strip image showing a random selection of areas

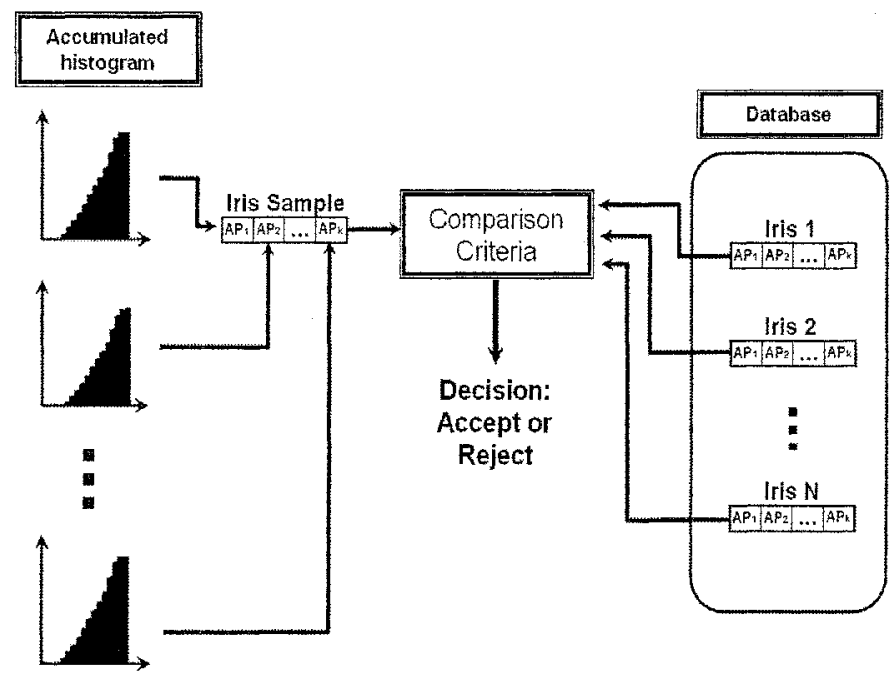

Fig. 5. The process of comparison and matching 


\section{Experiments}

Experiments were ran for a database with 210 samples coming from 70 individuals. In a first step, a set of experiments was scheduled to produce the best acceptance/rejection thresholds (Euclidean distance). In a second step, a set of 10 experiments were conducted by fixing both: the size of subimage area and the number of features or histograms. In every experiment a random iris sample was taken, and a random database of 100 samples was formed without duplicates. The percent of successful identifications reported is the mean of those 10 experiments. The Fig. 8 shows the accuracy on the accept/reject decision taken. The best results of $97.18 \%$ were obtained with an square subimage area of 25 pixels and 210 features. False positive errors were $0.26 \%$ and false negatives errors were $2.56 \%$.

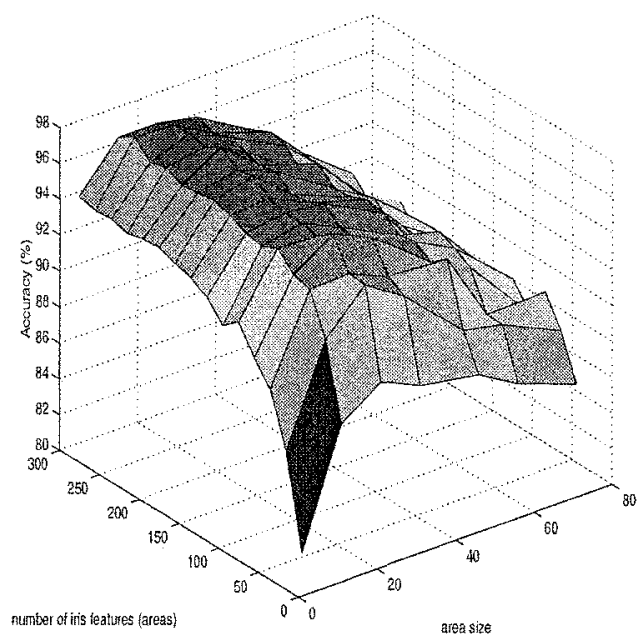

Fig. 6. Results from experiments with different number of features and size of areas

The Fig. 9 (a) shows the distribution observed in function of two types of persons, "authentics" and "impostors". This distribution estimation treats only one-to-one verification mode. We can observe a significant overlapping area, which in turns avoid more accurate scores of recognition. In Fig. 9 (b) is shown the ROC curve that is a plot of genuine acceptance rate against false acceptance rate. Points in the curve denote all possible system operating states in different tradeoffs. Fig. 9 (b) shows an acceptable performance of our method. 


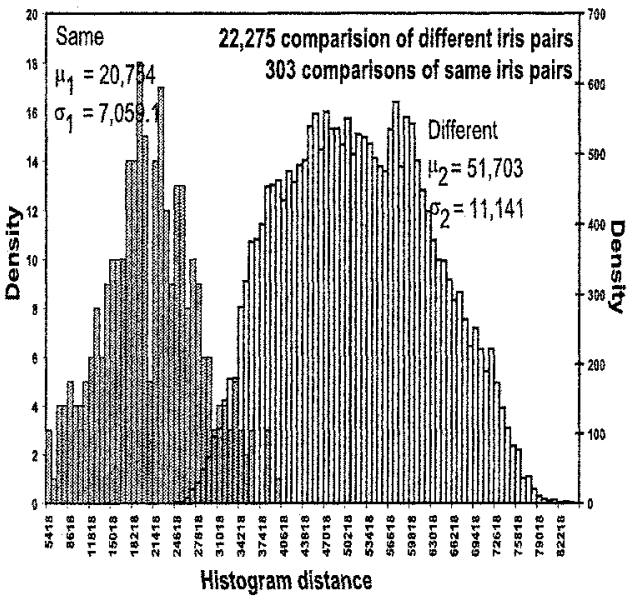

(a)

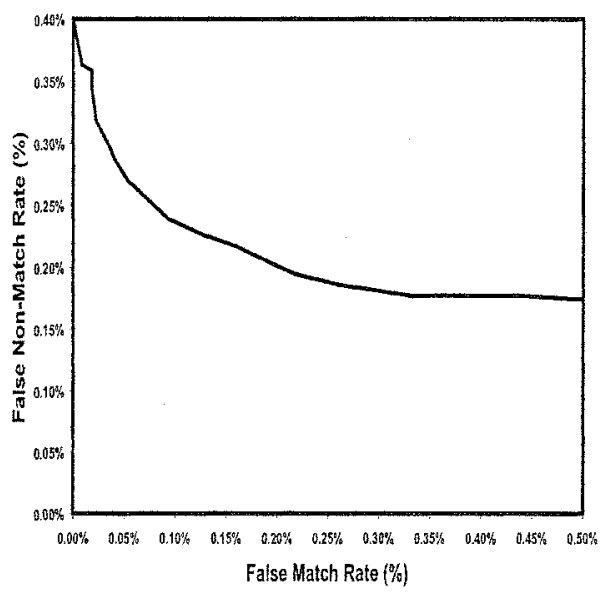

(b)

Fig. 7. (a) Probability Distribution of accumulated histograms distance for authentic persons (left) and impostors (right). (b) Receiving Operating Characteristic curve

\section{Conclusions and Future Works}

A new approach for iris recognition has been presented. The novel contribution relies on the feature characterization of iris by the use of a sampling technique and accumulated histograms. We work directly with the iris information, instead of using a bank of filters or making a mathematical transformation. Both approaches can be very computationally demanding. We claim than our approach will conduct to a fast approach for iris recognition, where just a few samples will be needed to discard many samples in database and allow a more focused sampling in a reduced set of candidates. In our proposal iris image is sampled in a specific number of randomly selected square areas or subimages. From every subimage an accumulated histogram is built, and the whole iris is represented by a set of accumulated histograms. The matching step consists then in a comparison between histograms, and the decision to accept or reject a user is taken based on the minimum difference between iris samples, and a threshold calculated experimentally.

We have found a rate of successful identifications in the order of $97 \%$.

In future works we will address two aspects: first, the determination of minimal number of samples and the size of samples, to identify accuratelly a person, and second, we are looking a more uniform sampling strategy to avoid redundant information by overlapping of samples. 


\section{References}

1. W. Boles and B. Boashash, "Iris Recognition for Biometric Identification using dyadic wavelet transform zero-crossing", IEEE Transactions on Signal Processing, Vol. 46, No. 4, 1998, pp. 1185-1188.

2. D. Clausi and M. Jernigan, "Designing Gabor Filters for Optimal Texture Separability", Pattern Recognition, Vol. 33, 2000, pp. 1835-1849.

3. J. Daugman, "How Iris Recognition Works", IEEE Transactions on Circuits and Systems for Video Technology, Vol. 14, No. 1, 2004, pp. 21-30.

4. M. Dobes, I. Machala, P. Tichasvky, and J. Pospisil, "Human Eye Iris Recognition Using The Mutual Information", Optik, No. 9, 2004, pp. 399-404.

5. Y. Huang, S. Luo, and E. Chen, "An Efficient Iris Recognition System", In Proceedings of the First International Conference on Machine Learning and Cybernetics, 2002 , pp. 450-454.

6. J. Huang, Y. Wang, T. Tan, and J. Cui, "A New Iris Segmentation Method for Iris Recognition System", In Proceedings of the 17th International Conference on Pattern Recognition, 2004, pp. 554-557.

7. A. Jain, R. Bolle, S. Pankanti, Biometrics: Personal Identification in Networked Society, Kluwer Academics Publishers, 2002.

8. A. Jain, A. Ross, A. Prabhakar, "An Introduction to Biometric Recognition", IEEE Transactions on Circuits and Systems for Video Technology, Vol. 14, No. 1, 2004, pp. 4-20.

9. L. Ma, Y. Wang, T. Tan, and D. Zhang, "Personal Identification Based on Iris Texture Analysis", IEEE Transactions on Pattern Analysis and Machine Intelligence, Vol. 25, No. 12, 2003, pp. $1519-1533$.

10. D. de Martin-Roche, C. Sanchez-Avila, and R. Sanchez-Reillo, "Iris Recognition for Biometric Identification using dyadic wavelet transform zero-crossing", In Proceedings of the IEEE 35th International Conference on Security Technology, 2001, pp. 272-277.

11. M. Negin, Chmielewski T., Salganicoff M., Camus T., Cahn U., Venetianer P., and Zhang G. "An Iris Biometric System for Public and Personal Use ", Computer, Vol. 33, No. 2, 2000, pp. 70-75.

12. Ch. Tisse, L. Martin, L. Torres, and M. Robert, "Person Identification technique using Human Iris Recognition", In Proceedings of the 15th Intemational Conference on Vision Interfase, 2002.

13. R. Wildes, "Tris Recognition: An Emerging Biometric Technology", Proceedings of the IEEE, Vol. 85, No. 9, 1997, pp. 1348-1363.

14. Y. Zhu, T. Tan, and Y. Wang, "Biometric Personal Identification Based on Iris Patterns", In Proceedings of the 15th International Conference on Pattern Recognition, 2000, pp. 801-804. 